\title{
Cirurgia valvar na infância: um novo aspecto de ação
}

José Teles de MENDONÇA*, José WANDERLEY NETO**, Marcos R. CARVALHO*, Rika K. da COSTA* $^{*}$, Edson FRANCO FILHO*, Geodete Batista COSTA*, Marcus Antônio L. de BARROS*, Clovis Oliveira ANDRADE*, Maria Luíza Dória ALMEIDA*, Lívia Chagas de BARROS*

MENDONÇA, J. T.; WANDERLEY NETO, J.; CARVALHO, M. R.; COSTA, R. K.; FRANCO FILHO E.; COSTA, G. B.; BARROS, M. A. L.; ANDRADE, C. O.; ALMEIDA, M. L. D.; BARROS, L. C. - Cirurgia valvar na infância: um novo aspecto de açāo. Rev. Bras. Cir. Cardiovasc., 7(3):174-179, 1992.

RESUMO: De janeiro de 1978 a dezembro de 1991, 148 crianças (idade igual ou menor a 15 anos) foram operadas pela primeira vez, para correçāo de defeitos adquiridos (doença reumática) em uma das valvas cardíacas. A casuística foi dividida em duas épocas, demarcadas pela determinação do Serviço em preservar o maior número possível das valvas tratadas, em especial, nesse grupo de pacientes. No último ano, foram operadas 24 crianças, nas quais a valva mitral foi abordada 20 vezes e a aórtica 8 vezes. O índice de aproveitamento da valva mitral foi de $95 \%$ ( $28 \%$ no período anterior) e da valva aórtica $62 \%$ ( $20 \%$ no período anterior). A mortalidade global foi de $8 \%$ no período anterior e nāo houve óbitos no último ano da experiência. Nenhum paciente apresentou qualquer complicaçāo e todos evoluíram satisfatoriamente. Concluímos que, com determinaçāo, é possível preservar a maior parte das valvas acometida por doença reumática na infância e sugerimos que a indicaçāo cirúrgica deva ser o mais precoce possível, para evitar alteraçōes no aparelho valvar secundárias à disfunçăo, bem como um seguimento tardio efetivo mais duradouro, para julgar se a plastia é realmente superior à substituiçāo valvar em pacientes jovens com doença reumática.

DESCRITORES: valvas cardíacas, cirurgia, crianças.

\section{INTRODUÇĀO}

Um grande número de crianças com idade menor ou igual a 15 anos é operado constantemente, em nosso Serviço ( $20 \%$ do movimento valvar).

Preocupados com os resultados tardios com uso de prótese $^{\star}$, e principalmente, influenciados pelos resultados conseguidos por ANTUNES et alii. ${ }^{1}$, na África do Sul, e, no Brasil, por GREGORI Jr. et alii. ${ }^{16}$, RIBEIRO et alii. ${ }^{21}$, BROFMAN ${ }^{7}$, ELOY et alii. ${ }^{14} \mathrm{e}$ LOBO FILHO et alii. ${ }^{18}$ que mostraram, claramente,

* WANDERLEY NETO et alii. - Substituição valvar em crianças até 15 anos: resultados em 149 casos. Trabalho apresentado ao $15^{\circ}$ Congresso Nacional de Cirurgia Cardíaca. Rio de Janeiro, RJ., 1988. ser possível reparar valvas reumáticas, decidimos entrar com determinação no programa, com o objetivo de preservar o maior número de valvas cardíacas, em especial nesse grupo seleto de pacientes.

O presente trabalho pretende mostrar essa extraordinária transformação.

\section{CASUÍSTICA E MÉTODOS}

De janeiro de 1978 a dezembro de 1991, 148 crianças (idade menor ou igual a 15 anos) foram operadas pela primeira vez, para correção de defeitos adquiridos (doença reumática) em uma das valvas cardíacas. A casuística foi dividida em dois grupos

\footnotetext{
Trabalho realizado na Unidade Cardiotorácica de Sergipe, Hospital de Clínicas Dr. Augusto Leite e Hospital Sảo Lucas. Aracajú, SE, Brasil. Apresentado ao $19^{\circ}$ Congresso Nacional de Cirurgia Cardíaca. Sảo Paulo, 7 a 9 de maio, 1992.

* Da Unidade Cardiotorácica de Segipe.

* Do Instituto de Doenças do Coraçảo da Santa Casa de Maceió, Maceió, AL.

Endereço para separatas: José Teles de Mendonça. Av. Francisco Porto, 1882, Apto. 1001. 49.000 Aracajú, SE, Brasil
} 
MENDONÇA, J. T.; WANDERLEY NETO, J.; CARVALHO, M. R.; COSTA, R. K.; FRANCO FILHO, E.; COSTA, G. B.; BARROS, M. A. L.; ANDRADE, C. O.; ALMEIDA, M. L. D.; BARROS, L. C. - Cirurgia valvar na infância: um novo aspecto de açảo. Rev. Bras. Cir. Cardiovasc., 7(3):174-179, 1992.

(Tabela 1), para evidenciar a diferença marcante na abordagem cirúrgica dessas crianças, a partir de 1991, quando o grupo optou por uma ação determinada na preservação dos elementos valvares. Durante - último ano (1991), foram operados, consecutivamente, 24 crianças com idade variando entre 5 e 15 anos (média de 12,5 anos). Quatorze $(58,3 \%)$ pertenciam ao sexo feminino e $10(41,7 \%)$ ao sexo masculino. Dezesseis $(66,6 \%)$ pacientes apresentavam lesão isolada na valva mitral (insuficiência 11, estenose 4 e dupla lesão 1). Quatro $(16,7 \%)$ pacientes tinham lesão isolada na valva aórtica, todos com insuficiência e em $4(16,7 \%)$ havia, concomitantemente, lesão nas valvas mitral e aórtica (insuficiência mitral + insuficiência aórtica 2 e dupla lesão mitral + insuficiência aórtica 2).

O diagnóstico foi sempre feito com base na ecodopplercardiografia e no mapeamento de fluxos a cores.

Todos os pacientes foram operados através de esternotomia mediana, com o auxílio da circulação extracorpórea convencional, de hipotermia moderada a $30^{\circ} \mathrm{C}$ e cardioplegia sangüínea isotérmica* intermitente injetada na raíz da aorta ou diretamente nos óstios coronarianos, quando houvesse insuficiência valvar.

A técnica cirúrgica na reparação valvar foi bastante variada e foi escolhida, a depender do aspecto morfológico e funcional da valva tratada.
A valva mitral foi abordada cirurgicamente 20 vezes (insuficiência 13, estenose 4 e dupla lesão 3 ) e pôde ser preservada em $19(95 \%)$ pacientes. Foi substituída em um único paciente. Utilizaram-se, basicamente, os preceitos de CARPENTIER ? com algumas variaçōes. Dessa forma, foram realizadas: comissurotomia em 8 pacientes, papilarotomia em 3 , encurtamento de cordas da cúspide anterior em 10 , ( 8 pela técnica de CARPENTIER ${ }^{\circ} \mathrm{e} 2$ pela técnica de GREGORI Jr. et alii ${ }^{15}$, secção de cordas secundárias da cúspide posterior em 4 e anuloplastia em 13 pacientes (11 com fita de pericárdio** e $2 \mathrm{com}$ anel $\left.\left.\right|^{\star \star \star}\right)$.

A valva aórtica foi abordada em 8 pacientes, todos com insuficiência valvar importante. Foi reparada em $5(62,5 \%)$ pacientes e substituída em $3(37,5 \%)$. O procedimento mais utilizado foi a anuloplastia comissural ${ }^{8}$, empregada em 4 pacientes. Em 2 pacientes a válvula não coronariana foi ampliada $(1 \mathrm{com}$ interposição de retalho de pericárdio bovino entre o anel e a base da válvula ${ }^{5} \mathrm{e} o$ outro ampliando-se a borda livre ${ }^{20}$.

O resultado do procedimento reparador foi avaliado pelo aspecto visual do aparelho valvar, tendo-se como base abertura, mobilidade e coaptação dos seus componentes, seguindo-se teste anti-refluxo. Para a valva mitral, injetava-se solução salina em ventrículo esquerdo, através da sonda $12 \mathrm{~F}$ introduzida via aorta, enquanto, pela atriotomia, se observava o desempenho valvar mitral. A condição da valva aórtica

TABELA 1

CIRURGIA VALVAR NA INFÂNCIA: DADOS COMPARATIVOS

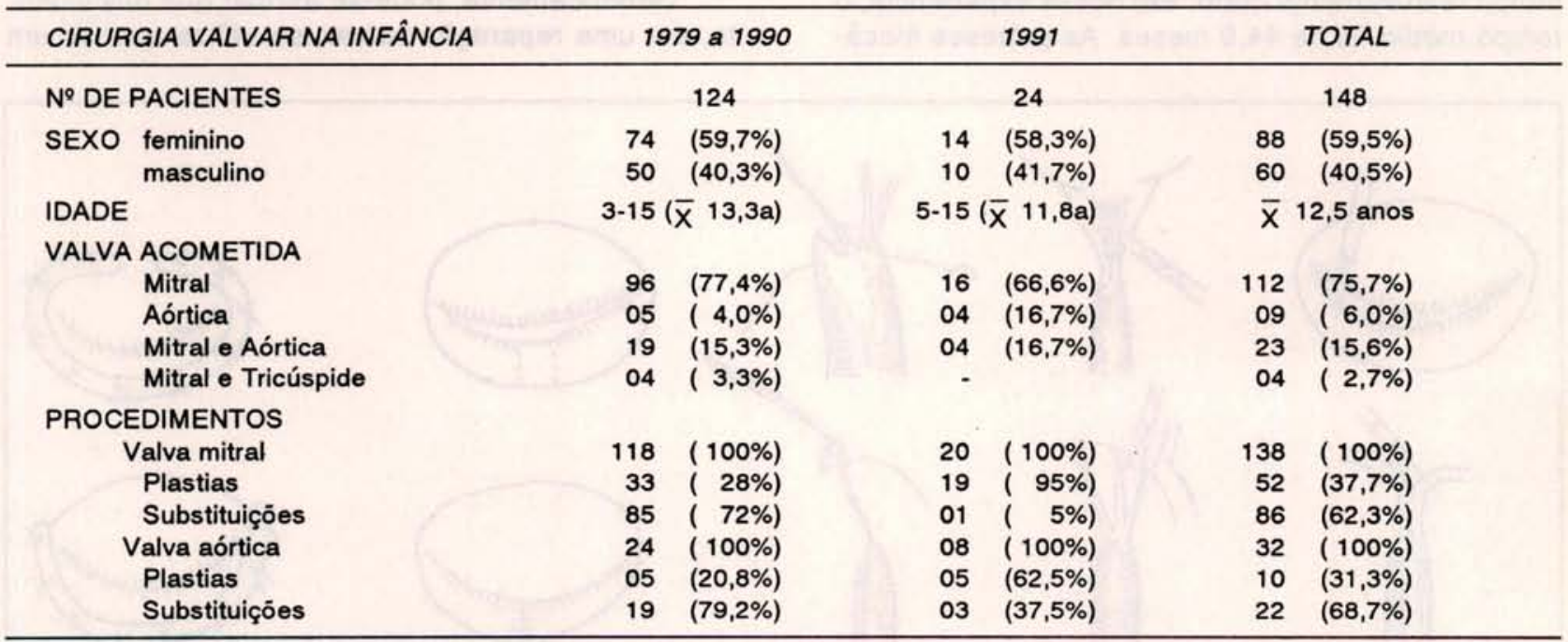

* Cardioplegia sangũínea realizada com o sangue na temperatura do perfusato.

* JATENE, A. D. - Comentário feito durante mesa-redonda, no $16^{\circ}$ Congresso Nacional de Cirurgia Cardíaca. São Paulo, SP., 1989.

* Anel modelo Carpentier, confeccionado pela Labcor com núcleo fino de Celcon revestido com pericárdio bovino. 
MENDONÇA, J. T.; WANDERLEY NETO, J.; CARVALHO, M. R.; COSTA, R. K.; FRANCO FILHO, E.; COSTA, G. B.; BARROS, M. A. L.; ANDRADE, C. O.; ALMEIDA, M. L. D.; BARROS, L. C. - Cirurgia valvar na infância: um novo aspecto de açāo. Rev. Bras. Cir. Cardiovasc., 7(3):174-179, 1992.

foi avaliada pela ausência de refluxo, observada visualmente para o ventrículo esquerdo, nos casos de abordagem simultânea da valva mitral ou pela ausência de distenção do ventrículo esquerdo após despinçamento da aorta, nos casos em que a valva aórtica foi abordada isoladamente.

\section{RESULTADOS}

A mortalidade hospitalar foi de $8,0 \%$ nos 124 pacientes operados entre 1978 a 1990; não houve óbitos entre os 24 operados em 1991. Nesse grupo, nenhum paciente apresentou complicaçāo significativa no pós-operatório e todos deixaram o hospital entre $\circ 8^{\circ}$ e o $10^{\circ}$ dia. Antes da alta, os pacientes foram submetidos a estudo ecodopplercardiográfico e nenhuma disfunção importante foi registrada, a nāo serum discreto refluxo, sem importância hemodinâmica, observado em alguns pacientes.

As crianças vêm sendo seguidas rigorosamente (tempo médio 7,8 meses) e, até o momento, nāo foi observada falência no procedimento reparador.

\section{COMENTÁRIOS}

As elevadas taxas de complicaçōes encontradas na evoluçāo de pacientes jovens submetidos a substituição valvar utilizando próteses têm causado preocupação e inquietação em todos os grupos cirúrgicos. As biopróteses, quando utilizadas nessa faixa etária, sofrem um processo rápido de degeneraçāo e calcificação, obrigando as crianças a reoperação em tempo relativamente curto. Em nossa experiência, o tempo médio foi de 44,9 meses. As próteses mecâ- nicas, embora resistentes, quando utilizadas em jovens sāo acompanhadas de maior morbidade, fenômenos tromboembólicos, ou complicaçōes decorrentes do uso obrigatório de anticoagulantes ${ }^{22}$.

Com a divulgação dos conceitos reparatórios de CARPENTIER ${ }^{\circ}$, aos grupos cirúrgicos foram incorporando essa metodologia e desenvolvendo novas idéias, de maneira que um número cada vez maior de pacientes pode ter suas valvas cardíacas preservadas. Os horizontes foram ampliados, não só pela introdução de novos procedimentos técnicos reparadores, como também pela abertura no legue das indicaçōes. Dessa forma, hoje podem ser preservadas as quatro valvas cardíacas, com as mais variadas causas de disfunção. Săo reparados defeitos valvares congênitos, doença degenerativa e até mesmo seqüelas de doenças inflamatórias ou infecciosas, como doença reumática e endocardite.

Os procedimentos reparadores da valva mitral foram introduzidos na quase totalidade por CARPENTIER $^{\circ} \mathrm{e}$, posteriormente, ampliados com algumas novas idéias ou modificaçōes nos conceitos préestabelecidos, assim resumidos: 1) procedimentos que visam aumentar a abertura e a mobilidade dos elementos valvares (comissurotomia, papilarotomia, fenestraçāo e secçāo de cordas secundárias; 2) procedimentos que visam aumentar a coaptaçāo: a) em relação às cordas tendíneas: encurtamento de cordas alongadas, translocaçāo de cordas, confecção de neocordas e implante com material biológico ou sintético; b) em relação às cúspides: ampliação ou ressecçāo parcial; c) em relação ao anel: anuloplastia). A Figura 1 mostra alguns desses procedimentos.

Genericamente, pode-se afirmar que dificilmente, em uma reparação valvar, se utilize apenas um

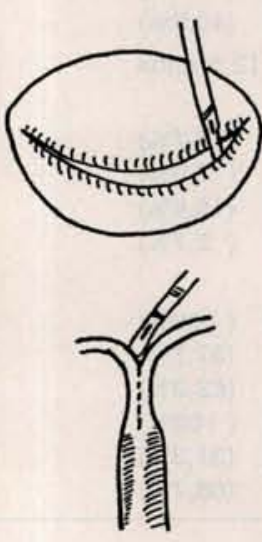

Comissurotomia Papilarotomia

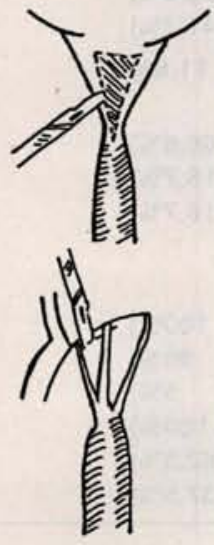

Fenestração Secçâo de Cordas

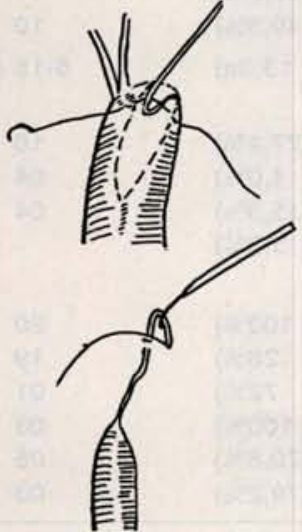

Encurtamento de Cordas
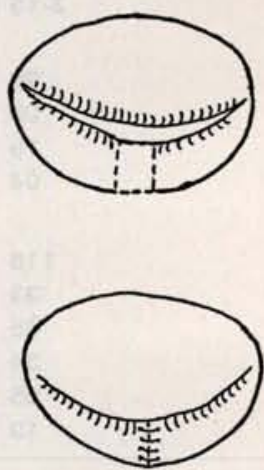

Ressecçáo Cúspide Posterior

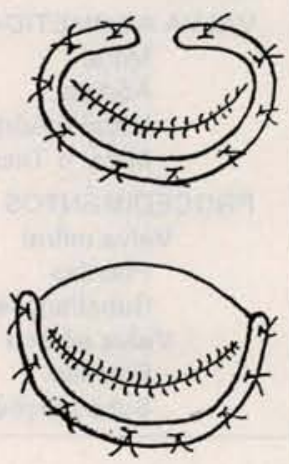

Remodelação do Anel

Fig. 1 - Cirurgia reconstrutiva da valva mitral. 
MENDONÇA, J. T.; WANDERLEY NETO, J.; CARVALHO, M. R.; COSTA, R. K.; FRANCO FILHO, E.; COSTA, G. B.; BARROS, M. A. L.; ANDRADE, C. O.; ALMEIDA, M. L. D.; BARROS, L. C. - Cirurgia valvar na infância: um novo aspecto de ação. Rev. Bras. Cir. Cardiovasc., 7(3):174-179, 1992.

desses procedimentos de forma isolada. Em nossos casos, tivemos oportunidade de usar muitas dessas técnicas com as mais variadas combinaçōes. Nos encurtamentos de cordas, empregamos, na maioria das vezes, a técnica do sepultamento no músculo papilar ${ }^{10} \mathrm{e}$, em alguns, a depender dos aspectos morfológicos ou facilidade técnica, realizamos encurtamento na cúspide anterior, como propōem GREGORI Jr. et alii ${ }^{15}$. Na remodelação anular, empregamos anel tipo Carpentier (anel confeccionado pela Labcor com núcleo fino de Celcon e revestido com pericárdio bovino preservado em glutaraldeído) ou fita de pericárdio bovino, como sugerem BRAILE et alii ${ }^{6}$, apenas na parte posterior do anel. Quando utilizamos a fita, não fazemos nenhuma medida, pois acreditamos que o pericárdio sofrerá maior ou menor enrugamento a depender da distância entre os fios dos pontos aplicados. Nesse caso, fica o senso artístico do cirurgião como o único referencial para aplicação dos pontos e posicionamento da fita. Além do mais, a técnica descrita por BRAILE et alii ${ }^{6}$ despreza a cúspide posterior que, ao nosso ver, após devidamente liberada, e em muitos casos, pode representar peça fundamental na composiçāo do aparelho valvar. Acreditamos que o uso do anel permite melhor padronizaçăo técnica, facilitando o ensino e, conseqüentemente, sua aplicaçāo em larga escala.

Além de todos os incovenientes da utilizaçāo das próteses em pacientes jovens, a reconstrução da valva mitral tem a grande vantagem de, preservando - aparelho subvalvar de sustentação, garantir a manutenção da funçăo ventricular ${ }^{12,17}$.

$\mathrm{Na}$ reconstrução funcional da valva aórtica, os procedimentos técnicos utilizados visam a: 1) aumentar a área de escoamento e permitir ampla movi- mentação das válvulas (comissurotomia e descalcificaçāo); 2) aumentar a área de contato entre as válvulas, melhorando a coaptação e evitando pontos de grande stress (anuloplastia: sutura contínua circular, e anuloplastia comissural ${ }^{8}$; ampliaçāo de válvulas retraídas: suturando-se pericárdio bovino à borda livre ${ }^{14}$, ou pericárdio tratado com glutaraldeído ${ }^{13}$, interpondo-se um retalho de pericárdio bovino entre $\circ$ anel e a base da válvula ${ }^{5}$; substituição de válvulas por homo-enxertos aórticos frescos ${ }^{19}$; suspensão de vávula ${ }^{24,26}$ e ressecção triangular $\left.{ }^{11}\right)$. A Figura 2 ilustra parte desses procedimentos.

Em nossa casuística, de 32 valvas aórticas tratadas em pacientes jovens (idade menor ou igual a 15 anos), $10(31,3 \%)$ foram restauradas. No último ano, de 8 valvas operadas, $5(62,5 \%)$ puderam ser submetidas a procedimentos reconstrutivos, evidenciando a grande determinação em preservar as valvas nativas e, principalmente, a possibilidade de conseguir.

A escolha do método reconstrutivo nem sempre é fácil e, por vezes, se faz necessária a utilizaçāo de manobras associadas. Empregamos a anuloplastia comissural quando não existe grande comprometimento estrutural da valva e as válvulas se encontram bem preservadas. Quando existe retraçăo fibrótica de um ou mais elementos, a técnica de escolha é substituiçāo ou ampliação da válvula comprometida e, nesses casos, realizamos, sempre, associadamente, a anuloplastia nas comissuras relacionadas à válvula corrigida. Acreditamos que a ampliaçăo da válvula permite melhor distribuição das forças, aumentando a possibilidade de melhores resultados não só a curto prazo, como mostram DURAN et alii ${ }^{13}$, mas também tardiamente.

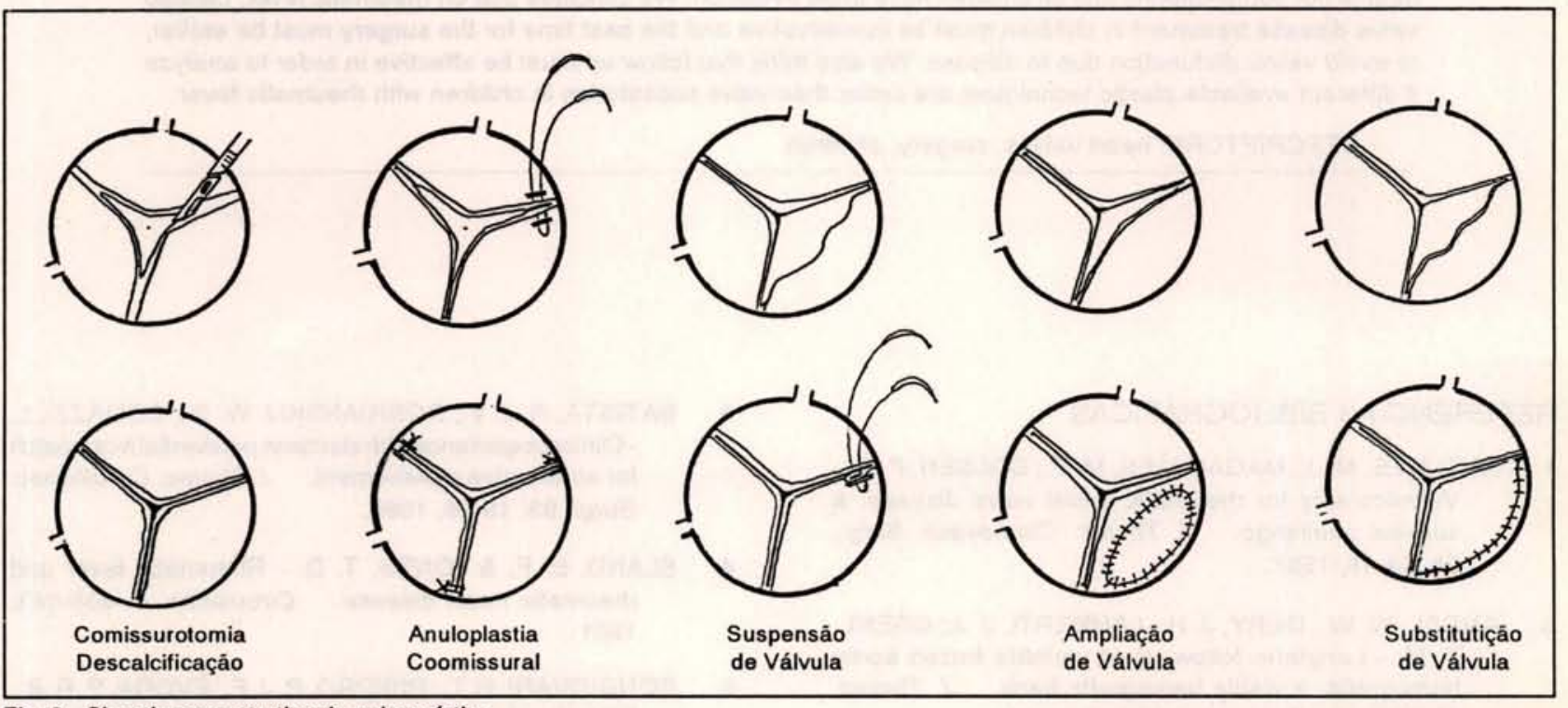

Fig. 2 - Cirurgia reconstrutiva da valva aórtica. 
MENDONÇA, J. T.; WANDERLEY NETO, J.; CARVALHO, M. R.; COSTA, R. K.; FRANCO FILHO, E.; COSTA, G. B.; BARROS, M. A. L.; ANDRADE, C. O.; ALMEIDA, M. L. D.; BARROS, L. C. - Cirurgia valvar na infância: um novo aspecto de açāo. Rev. Bras. Cir. Cardiovasc., 7(3):174-179, 1992.

A cirurgia reconstrutiva da valva aórtica tem algumas vantagens, em relação à utilizaçāo de próteses em pequenos pacientes: 1) menor gradiente transvalvar; 2) a ausência de suporte de sustentaçāo diminui o stress sobre o bio-enxerto utilizado, diminuindo a incidência de fenômenos degenerativos e aumentando o tempo de vida útil ${ }^{2}$; 3) possibilidade de crescimento anular mesmo quando toda uma válvula é substituída; para isso, BATISTA et alii ${ }^{3}$ recomendam a utilizaçăo de enxerto um pouco redundante; 4) preservando-se total ou parcialmente as válvulas, obtém-se maior confiabilidade, uma vezque as disfunçōes graves, quando ocorrem, costumam acontecer precocemente no período em que se tem completa vigilância sobre os pacientes e essas falhas podem ser corrigidas; tardiamente, a degradaçāo nunca vai ser catastrófica.

Por fim, nāo podemos esquecer que a doença reumática tem características próprias que podem interferir no sucesso do procedimento. Embora SHORE et alii ${ }^{23}$ tenham encontrado piores resultados com plastias, nas disfunçōes de etiologia reumática, a experiência tem demonstrado que valvas funcionalmente anormais, durante uma cardite reumática aguda, podem retornar ao normal, especialmente quando as recurrências sāo prevenidas ${ }^{4,25}$. Aquimioprofilaxia da recurrência com penicilina é obrigatória '.

\section{CONCLUSŌES}

Concluímos que, com determinação, é possível preservar a maior parte das valvas acometidas por doença reumática na infância e sugerimos que a indicaçăo deva ser o mais precoce possível, para evitar as alteraçōes no aparelho valvar secundárias à disfunção, bem como um seguimento tardio efetivo e duradouro, para julgar se a plastia é realmente superior à substituiçăo valvar em pacientes jovens com doença reumática.

MENDONÇA, J. T.; WANDERLEY NETO, J.; CARVALHO, M. R.; COSTA, R. K.; FRANCO FILHO, E.; COSTA, G. B.; BARROS, M. A. L.; ANDRADE, C. O.; ALMEIDA, M. L. D.; BARROS, L. C. - Valvular surgery in childhood: new trends. Rev. Bras. Cir. Cardiovasc., 7(3):174-179, 1992.

ABSTRACT: From January 1978 to December 1991, 148 children (age $\leqslant 15$ years) were operated upon on our Service for the first time, to correct defects (rheumatic fever) on the cardiac valves. The material was divided in two times, according to the point of view of the group in an attempt to preserve a great number of valves, in especial in children. Last year 24 children were operated upon in whom mitral valve disease treatment was necessary twenty times, and in aortic valve eight times. In that group, $95 \%$ (28\% before) surgery on the mitral valve and $62 \%(20 \%$ before) on the aortic valve were conservative procedures. There were neither deaths nor complications and all children have good evolution. We conclude that on rheumatic fever, cardiac valve disease treatment in children must be conservative and the best time for the surgery must be earlier, to avoid valvar disfunction due to disease. We also think that follow up must be effective in order to analyze if different available plastic techniques are better than valve substitution in children with rheumatic fever.

DESCRIPTORS: heart valves, surgery, children.

\section{REFERÊNCIAS BIBLIOGRÁFICAS}

ANTUNES, M. J.; MAGALHÄES, M. P.; COLSEN, P. R. Valvuloplasty for rheumatic mitral valve disease: a surgical challenge. J. Thorac. Cardiovasc. Surg., 94: $44-48,1987$.

ANGEL, W. W.; OURY, J. H.; LAMBERTI, J. J.; GREHL, T. M. - Longterm follow up of variable frozen aortic homografts: a viable hemografts bank. J. Thorac, Cardiovasc. Surg., 93: 815-822, 1987.
3 BATISTA, R. J. V.; DOBRIANSKIJ, W. G., COMAZZI, L. - Clinical experience with stentless pericardial monopatch for aortic valve replacement. J. Thorac. Cardiovasc. Surg., 93: 19-26, 1989.

4 BLAND, E. F. \& JONES, T. D. - Rheumatic fever and rheumatic heart disease. Circulation, 4: 836-843, 1951.

5 BONGIOVANI, H. L.; RIBEIRO, P.J. F.; ÉVORA, P. R. B.; BRASIL, J. C. F.; REIS, C. L.; SGARBIERI, R. N. - 
MENDONÇA, J. T.; WANDERLEY NETO, J.; CARVALHO, M. R.; COSTA, R. K.; FRANCO FILHO, E.; COSTA, G. B.; BARROS, M. A. L.; ANDRADE, C. O.; ALMEIDA, M. L. D.; BARROS, L. C. - Cirurgia valvar na infância: um novo aspecto de açảo. Rev. Bras. Cir. Cardiovasc., 7(3):174-179, 1992.

Plastia valvar aórtica por ampliação de válvula com pericárdio bovino: nota prévia. Rev. Bras. Cir. Cardiovasc., 3: 130-133, 1988.

BRAILE, D. M.; ARDITO, R. V.; PINTO, G. H.; SANTOS, J.L.V.;ZAIANTCHIC, M., SOUZA, D.R.S.;THEVENARD, R. - Plástica mitral. Rev. Bras. Cir. Cardiovasc., 5: 86-98, 1990.

BROFMAN, P. R. - Cirurgia conservadora da válvula mitral em pacientes jovens: técnica cirúrgica e resultados. São Paulo, 1986 [Tese. Doutorado. Faculdade de Medicina da Universidade de São Paulo].

CABROL. C.; GUIRAUDON, G.; BERTRAND, M. - Le traitement de l'insuffisance aortique par l'annuloplastie aortique. Arch. Mal. Coeur, 59: 1305-1312, 1966.

CARPENTIER, A. - Cardiac valve surgery: "The French Correction". J. Thorac. Surg., 86: 323-230, 1983.

CARPENTIER, A.; CHAUVAUD, S.; FABIANI, J. M. Reconstructive surgery of mitral incompetence: tenyear appraisal. J. Thorac. Cardiovasc. Surg., 79: 338-342, 1980.

COSGROVE, D. M.; ROSENKANZER, L. W.; HENDREN, W. G.; BARTLETT, J. L.; STEWART, W. J. - Valvuloplasty for aortic insufficiency. J. Thorac. Cardiovasc. Surg., 102: $571-577,1991$.

DAVID, T. E.; UDEN, D. E.; STRAUSS, H. D. - The importance of the mitral apparatus in left ventricular function after correction of mitral regurgitation. Circulation, 68 (Parte 2): 76-82, 1983.

DURAN, C.; KUMAR, N.; GOMETZA, B.; ALHALEES, Z. - Indications and limitations of aortic valve reconstruction. Ann. Thorac. Surg., 52: 447-455, 1991.

ELOY, R.; FERREIRA, Jr, A.; CARVALHO, H.; BRITO, J. C.; TADEU, E.; NERY, A. C.; RIBEIRO, A.; OLIVEIRA, R.; RIBEIRO, N. - Cirurgia orovalvar na criança: experiência de 135 casos. Rev. Bras. Cir. Cardiovasc., 1: 24-28, 1986.

GREGORI Jr, F.; TAKEDA, R.; FAÇANHA, L. - Nova técnica reconstrutiva na insuficiência valvar mitral por alongamento das cordas tendíneas da cúspide anterior. Arq. Bras. Cardiol., 54: 205-208, 1990.
GREGORY Jr, F.; TAKEDA, R.; SILVA, S. S.; - Sete anos de experiência com plastia da valva mitral. Arq. Bras. Cardiol., 47: 269-275, 1986.

17 LESSANA, A.; HARREMAN, F.; BOFFETYC, G. - Hemodynamic and cineangiographic study before and after mitral valvuloplasty (Carpentier's technique). Circulation, 64 (Parte 2): 195-202, 1981.

LOBO FILHO, G.; MARTINS, J. M.; OLIVEIRA, F. M.; MEMÓRIA Jr., J. M. F.; PIMENTA, F.; ARAÚJO, J. A. R.; ALENCAR, J. E. B.; BARBOSA, R.; LESSANA, A.; MAGALHĀES, O. - Cirurgia reconstrutiva da válvula mitral pelatécnica de Carpentier. Rev. Cir. Cardiovasc. 1: $16-18,1988$.

19 MARC, R.; KATZ, W. G.; GLENN, P. B.; DANNA, J.; RICHARD, L. - Homograft replacement of single aortic cusp: 22-year follow up. J. Cardiac. Surg., 3: 279$303,1988$.

20 MOHAMED, R.; AL FAGIH, F. R. C. S.; AL KASAB, M. R. C. P.; ASHMEG, F. R. C. S. - Aortic valve repair using bovine pericardium for cusp extension. J. Thorac. Cardiovasc. Surg., 96: 760-764, 1988.

21 RIBEIRO, E. J.; ALMEIDA, R. M.; FERREIRA, A. - Cirurgia reconstrutiva da válvula mitral. Arq. Bras. Cardiol., 41: 28-34, 1983.

SADE, R. M.; CRAWFORD, F. A.; FYFE, D. A.; STROUD, M. R. - Valve prostheses in children: a reassessment of anticoagulation. J. Thorac. Cardiovasc. Surg., 95: 553-561, 1988.

23 SHORE, D. F., WONG, P.; PANETH, M. - Result of mitral valvuloplasty with a suture plication technique. J. Thorac. Cardiovasc. Surg., 79: 349-357, 1980.

24 STARR, A.; MENASHE, V.; DOTTER, D. - Surgical correction of aortic insufficiency with ventricular septal defect. Surg. Gynecol. Obstet., 111: 71-76, 1960.

TOMPKINS, D. G.; BOXERBAUM, B.; LIEBMAN, J. Longterm prognosis of rheumatic fever patients receiving regular intramuscular benzatina penicillin. Circulation, 45: 543-551, 1972.

26 TRUSLER, G. A.; MOES, C. A. F.; KIDD, B. S. L. - Repair of ventricular septal defect with aortic insufficiency. J. Thorac. Cardiovasc. Surg., 66: 394-403, 1973. 